\title{
MULTIFUNCTIONAL REACTORS
}

\author{
K.R. Westerterp \\ Chemical Reaction Engineering Laboratories \\ Chemical Engineering Department \\ Twente University \\ P.O. Box 217, 7500 AE Enschede, the Netherlands
}

\begin{abstract}
Multifunctional reactors are single pieces of equipment in which, besides the reaction, other functions are carried out simultaneously. The other functions can be a heat, mass or momentum transfer operation and even another reaction. Multifunctional reactors are not new, but they have received much emphasis in research in the last decade. A survey is given of modern developments and the first successful applications on a large scale. It is explained why their application in many instances is still far-away from large scale introduction.
\end{abstract}

\section{INTRODUCTION}

A large research effort is put into the development of chemical reactors. In the last decade the demand for better selectivities and higher conversions has increased more than ever to ensure prevention of the release or removal of products - often in very minor quantities - that would otherwise harm our natural environment. Also, energy saving considerations have strongly influenced the choice of type and design of a reactor and have also lead to new, ingenious configurations and designs.

Also, in the last decade the so-called "multifunctional" reactors have received a strong developmental push. We owe the word "multifunctional reactor" to Agar and Ruppel (1988), who, in a pioneering paper, have given a survey of the possibilities and some practical examples of the application of the principle of multifunctionality. Its meaning is very simple: in a single piece of apparatus used for reaction, more than one function is executed. The other functions can be: mass, heat and momentum transport operations or even other, independent reactions.

Of course the principle of multifunctionality is not new and has been applied in many instances. Historically the oldest reactors were the stirred pots, and for catalytic processes the adiabatic bed. The retort was a multifunctional reactor combining reaction with mass transfer, distilling off a product. As soon as a cooling jacket or cooling coil was installed in the stirred pot we combined reaction with heat transfer. Also in our combustion engines, reaction and momentum transport are executed in the same cylinder. But today the degree of sophistication in using multifunctionality has increased by an order of magnitude.

We are not going to give a complete survey of the literature on multifunctional reactors nor a full outline of all the possibilities. We will only give a few examples to demonstrate the possibilities and challenges in the use of the principle of multifunctionality. We will discuss practical examples that are still at the research stage, or have already reached commercial application on an industrial scale. We will also, now and then, give a warning not to be over-optimistic; the reality of process economics puts strict limits on new developments. In placing limits we do not want to stop the researcher in his endeavours, but intend rather to stimulate him to make his invention even more efficient so that in the long run it can compete with existing reactors and eventually replace them. 
Reactor capacities, of course, vary widely. However, if one studies reactor capacity in certain branches of the process industries, some approximate ranges can be found. Thus, for the bulk chemical and oil industries capacities almost always vary between 4 to $50 \mathrm{kmoles}$ produced per $\mathrm{m}^{3}$ reactor volume per hour. The lower value relates to an economic boundary where reaction volume becomes too large and therefore uneconomical. The upper boundary refers to a technical limit: it becomes impossible to adequately withdraw the heat produced. For fine chemicals, where we mostly use batch processes, capacity levels are much lower and range from 0.1 to $1 \mathrm{kmol} / \mathrm{m}^{3} \mathrm{hr}$. For biotechnological processes capacities are also much lower and range from $1 \mathrm{kmol} / \mathrm{m}^{3} \mathrm{hr}$ for the low cost products to very low values of say $500 * 10^{-6} \mathrm{kmol} / \mathrm{m}^{3} \mathrm{hr}$ for high cost chemicals like penicillin. In such processes heat removal rates are no problem, but other operations may be limiting, e.g. oxygen supply may be the dominating factor determining capacity.

In nature, processes are even many decades slower, e.g. in petroleum geology, production rates range from $10-200 * 10^{-12} \mathrm{kmols}$ converted $/ \mathrm{m}^{3} \mathrm{hr}$.

In our discussion we are primarily interested in reactors for bulk chemicals and will now have a further look at multifunctional reactors, where reaction and heat transfer are combined.

\section{SIMULTANEOUS REACTION AND HEAT TRANSFER}

Historically we see that soon the kettle type reactors were equipped with cooling equipment, such as coils and wall jackets, in order to cope with the heat effects of chemical reactions. Temperature rises in adiabatic catalyst beds were kept generally under control by dividing the bed into several parts and installing coolers between them. Then injection of cold feed gases between catalyst beds was practised. For reactions with very high heat effects, the (cooled) tubular reactor was soon developed, despite mechanical difficulties in coping with thermal expansion of the apparatus during operation. Early applications of these reactors were oxidation and hydrogenation reactions, which are highly exothermic. We should note that in the oil industry, the tubular reactor for endothermic reactions had been introduced long ago: in thermal cracking furnaces the endothermic heat of reaction required was supplied directly in a furnace by combustion flames.

Later the good heat transfer and temperature equilibration properties of the fluid bed reactor were recognised and since then many fluid bed systems have been installed. Agar and Ruppel (1988) describe a clever use of the principle of multifunctionality, in which an inert fluidizable powder is blown through a stationary catalyst bed and there absorbs the reaction heat. After leaving the catalyst bed the heated powder is cooled and returned to the bottom of the bed, where it is again carried through the catalyst bed by the fresh feed gas. With high circulation hot spots can be suppressed effectively and the reactor operates almost isothermally, see also Saatdjian and Large (1985).

The removal of reaction heat by evaporation of a solvent is another technique, which has been applied several times and is used, e.g. in the oxidation of para-xylene using homogeneous catalysts in the wellknown Amoco process. The solvent is condensed outside the reactor and recycled. Due to mixing hy the gas flow, these reactors behave like a tank reactor. Van Gelder et al. (1990a, 1990b) have discussed a more sophisticated application of cooling by evaporation, this time in a packed bubble column, which is packed with solid catalyst particles. The gas and liquid flow upward through the column, at the top the liquid and vapour-gas mixtures are separated. The principle of multifunctionality here gives us several significant advantages: $1^{\circ}$ No expensive or elaborate cooling systems are required in the catalyst bed, $2^{\circ}$ the reactor pressure can be set and hence, the maximum temperature is easily controlled, $3^{\circ}$ temperature runaways are impossible because further heat production only increases the evaporation rate, $4^{\circ}$ because of the packing, axial dispersion is suppressed, so that plug flow is approached in longer, slender columns. They recommend the method of hydrogen starvation, in cases, where hydrogenation has to be carried out to produce selectively an intermediate product in a consecutive reaction system. This combination of reaction with evaporation also can easily cope with a deactivating catalyst and Westerterp et al. (1988) specially recommend this reactor type with evaporative cooling for application to catalytic hydrogenations in the fine chemicals industries. Compared with a more classical reactor, the partial pressure of hydrogen, and therefore the reaction rate, is reduced due to the vapour pressure of the solvent, but this is more than off set by the ease of heat removal and temperature control. 
Adiabatic bed reactor with periodic flow reversal

About 25 years ago in Novosibirsk, Boreskov et al. (1979) started the development of the adiabatic bed reactor with periodic flow reversal (RPFR) and its development was brought to completion by Matros $(1985,1989)$ during the eighties. The principle was developed aimed at a simplification of chemical process equipment and specially at a reduction of the use of high quality steel in the Sovjet Union, required for industrial scale manufacture of ammonia and sulphuric acid.

As this was not a problem in other parts of the world, it remained a local development. Later, however, it was recognised that the RPFR had great advantages in better pollution control and so Eigenberger and coworkers $(1988 \mathrm{a}, 1988 \mathrm{~b}, 1991)$ started to develop this multifunctional reactor for the catalytic combustion of volatile organic compounds in exhaust air. We will describe its behaviour on the basis of the oxidation of combustibles present in low concentration in air.

The RPFR works as follows. If the catalyst mass is hot, a cold air stream, containing some combustibles, will be heated up by the hot catalyst. Simultaneously the catalyst mass is cooled down. The air stream being heated up at some place in the catalyst bed will reach the temperature at which the combustibles ignite and the combustion reaction starts. Now, the combustion heat liberated will heat up the solids phase. If in the area of the catalyst bed outlet there is a zone where the catalyst is cold, the hot gas will heat up this cold catalyst. So in this part of the bed a heat wave will travel through the bed towards the outlet. And simultaneously near the inlet, where the hot bed is being cooled down, a cold wave is generated. In the zone between the two waves the bed is hot and here the combustibles are burnt.

By periodically reversing the direction of flow, see Fig. 1, heat can be kept in the reactor and by so doing the temperature in the middle zone will remain high and above the ignition temperatures. To reach a steady

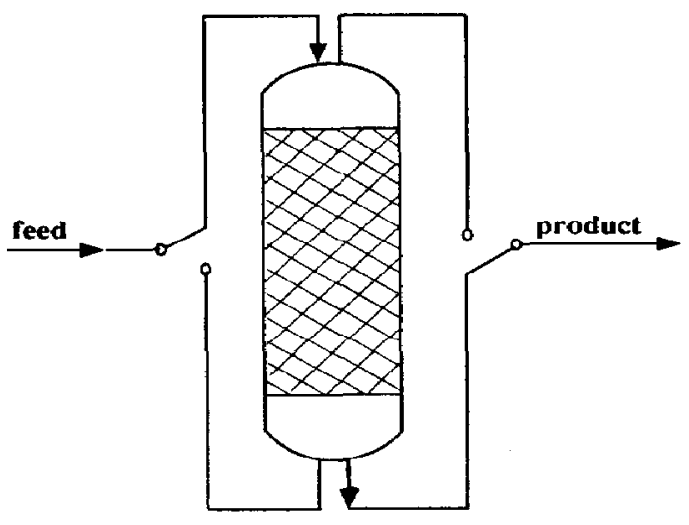

Fig. 1. The adiabatic bed reactor with periodic flow reversal. partially as a heat exchanger, to heat up and cool down contaminated air, and partially as a combustion reactor. The plateau temperature of the hot zone is influenced by many variables. The concentration of combustibles in the air must never be so high that the oxidation catalysts become overheated and loose their activity.

The RPFR therefore operates under dynamic conditions. The hot and cold fronts move back and forth with cycle time an important operating variable in preventing blow out of the reactor. The inlet and outlet zones act as heat exchangers and even at very low adiabatic temperature rises, $\Delta \mathrm{T}_{\text {sd }}$, due to very low concentrations of combustibles, heat exchange is so effective that the actual temperature rise is many times higher than $\Delta T_{\text {ad }}$. Further, the switch valves operate at low temperatures, which increases long service life.

For expensive catalysts the parts of the bed in heat exchange service can be replaced by inert material. We observe that in the RPFR expensive gas-gas heat exchangers are replaced by an additional layer of inert material installed in the same reactor shell. 


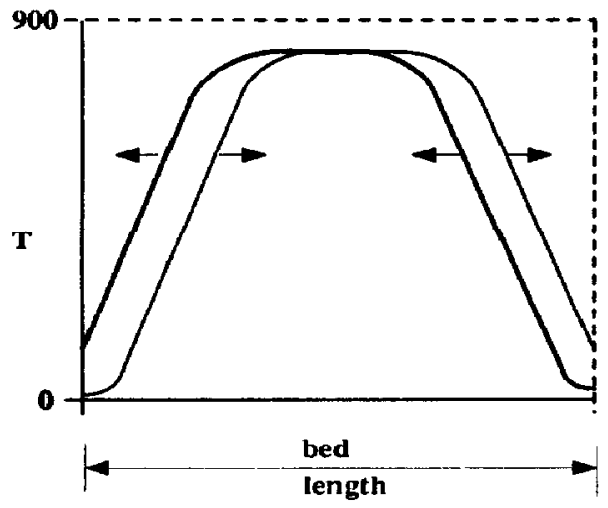

Fig. 2. Temperature profile just before reversal of the flow

For ammonia synthesis and $\mathrm{SO}_{2}$ oxidation, the RPFR has the advantage that towards the outlet the temperatures drops, meanwhile the reaction continues in the first part of the cold section. For exothermic equilibrium reactions this leads to somewhat higher conversions than in the usual commercial reactors. A disadvantage is that on reversing the flow the non-reacted materials in the bed are now purged into the outlet stream, so reducing the conversion efficiency. This can not be avoided, but for long cycle times the effect is negligible.

In the two examples given, of combined reaction and heat transfer in a multifunctional reactor, with either an evaporating solvent or with periodic flow reversal, both will have the same or higher reactor capacity than their classical counterparts, provided that also the volume of the heat exchange equipment is taken into account.

After these examples we will now refer to multifunctional reactors which incorporate mass transfer in the reaction zone.

\section{SIMULTANEOUS REACTION AND SEPARATION BY MASS TRANSFER}

The combination of reaction and separation by mass transfer is not new and many lucid examples can be found in the history of chemical engineering. We do not mean heterogeneous reactions, where mass transfer and reaction are combined in a multiphase reactor system, but refer to reactors in which in the same apparatus one of the reaction components is removed or supplied by a mass transfer separation process. We will consider two older examples of combinations with extraction and distillation.

Schoenemann and Hofmann (1957) elaborated a process to make furfural from xylose in aqueous solution containing $\mathrm{HCl}$. The furfural, which is produced in a combination of parallel and consecutive reactions, can decompose into resins and condensation products, so that yields of only around $10 \%$ can be expected. Now, by adding tetraline as an immiscible solvent phase they extracted the furfural as it was produced and obtained yields of over $60 \%$ on xylose!

Geelen and Wijfels (1965) studied the equilibrium transesterification of vinyl acetate and stearic acid to vinyl stearate and acetic acid. As a byproduct ethylidine acetate was formed. By executing this reaction in a distillation column they removed acetic acid selectively as soon as it had been formed, and by doing so suppressing the formation of the byproduct almost completely. Moreover, by removing the acetic acid, they could bring the equilibrium reaction to completion with a very high selectivity! We now will discuss more recent developments in the field of multifunctional reactors, namely the chromatographic reactor, catalytic distillation reactors, membrane reactors and the application of multifunctionality to the methanol synthesis.

The chromatographic reactor

The chromatographic reactor has already received attention for several decades, see Coca and Langer (1983). It is well known, that e.g. in an equilibrium reaction such as $A \leftrightarrows B$, where the two 
components are adsorbed with differing strengths, that a pulse of $A$ injected into an inert carrier stream flowing over a catalytically active adsorbent, will result in conversion to $B$ beyond the equilibrium position. This occurs if $A$ is strongly adsorbed and $B$ not, the column is long enough and the reaction is slow. As soon as some adsorbed $A$ is converted, the $B$ produced is carried away by the inert gas, so keeping the concentration of $B$ low in the vicinity of adsorbed $A$.

Aris and coworkers, see Fisch et al. (1986) and Ray et al. (1990), have adapted this principle very elegantly to a continuous process. Pure $A$ is injected somewhere in the middle of a column, through which a solid adsorbent-catalyst move slowly downwards and an inert carrier gas flows upwards. A (in an equilibrium reaction) is converted into $B$ which is less strongly adsorbed. Now the downward flow rate can be adjusted such that $B$ moves upwards and $A$ downwards. Experimentally they studied the hydrogenation of mesitylene to trimethylcyclohexane TMC. Under their conditions they obtained a top product of $90 \%$ TMC, which was above the equilibrium composition.

The operation of a moving bed reactor is delicate and advantages must be sufficiently great to have an incentive for developing the moving bed chromatographic reactor for large scale operation. Agar and Ruppel (1988) in an elegantly novel approach used the principle of the periodic flow reversal to avoid the moving bed operation. They applied the principle to the removal of nitrogen oxides from combustion gases:

$$
\begin{aligned}
& \mathrm{NH}_{3}+\text { adsorbent } \rightarrow \mathrm{NH}_{3} \text { ads } \\
& \mathrm{NH}_{3 \text { ads }}+\mathrm{NO}+0.25 \mathrm{O}_{2} \rightarrow \mathrm{N}_{2}+1.5 \mathrm{H}_{2} \mathrm{O}+\text { adsorbent }
\end{aligned}
$$

In commercial steady state processes it is impossible to supply the correct stoichiometric amount of $\mathrm{NH}_{3}$ to remove the $\mathrm{NO}$. $\mathrm{NH}_{3}$ is not allowed into the atmosphere and therefore only $80-90 \%$ of the required amount is fed to the reactor to allow for fluctuations in concentration and flow rates. Agar and Ruppel feed combustion gases to the Denox reactor and periodically give a large shot of $\mathbf{N H}_{3}$ to the feed stream. The overdosed $\mathrm{NH}_{3}$ reacts with the NO and the large excess is adsorbed on the Denox catalyst. An adsorption wave thus will travel through the catalyst bed in the direction of flow. After a certain period, the supply of $\mathrm{NH}_{3}$ is stopped and the reaction continues, consuming the adsorbed $\mathrm{NH}_{3}$. So a reaction wave also travels through the reactor. Before the NO breaks through, the direction of flow is reversed, and a new pulse of $\mathrm{NH}_{3}$ is supplied at the inlet, again increasing the amount of $\mathbf{N H}_{3}$ absorbed. The adsorption front now travels in the other direction, and as soon as the $\mathrm{NH}_{3} \mathbf{p u l s e}$ is stopped, a second reaction front will also follow. By periodically changing the direction of the flow, the $\mathrm{NH}_{3}$ is kept within the reactor and break-through is prevented. Furthermore a complete conversion of NQ is now achieved and fluctuations in the concentrations are now coped with, by the buffering capacity of the catalyst. In the work of Matros periodic flow reversal is applied to a heat transfer situation, whereas in the work of Agar and Ruppel it is a mass transfer application. Their process promises to become the first large scale application of the chromatographic reactor.

\section{The catalytic distillation reactor}

The combination of reaction and distillation over a catalyst bed has been introduced to the large scale manufacture of methyl-tertiary butyl ether MTBE, see Smith and Huddleston (1982). To produce MTBE, isobutylene is reacted with methanol, see Fig. 3. The separation is improved by forming a

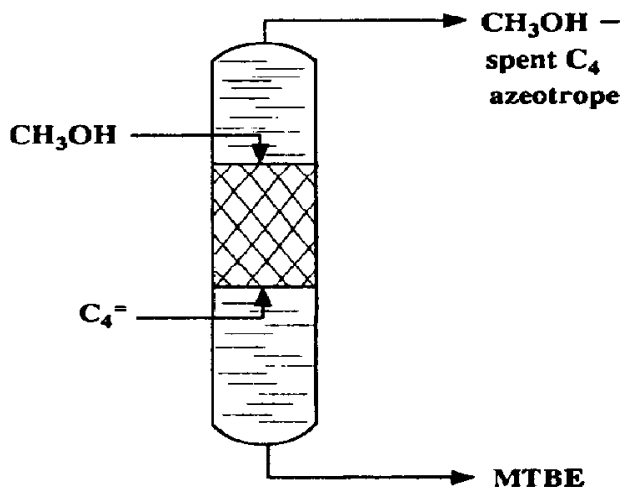

Fig. 3. Catalytic destillation. 
methanol-MTBE azeotrope. Pure MTBE leaves from the bottom of the reaction-distillation column. From the top, a methanol- $\mathrm{C}_{4}$ azeotrope is distilled. The catalyst used is an ion exchange type resin, which requires low reaction temperatures.

Several advantages are apparent. The heat of reaction is used completely in the distillation, so saving on steam consumption is achieved. The methanol is fed to the top of the catalyst bed, where the isobutylene concentrations are low, and the high $\mathrm{CH}_{3} \mathrm{OH}$ concentration pushes the equilibrium towards MTBE. Further, the MTBE is removed more or less directly after it has been formed. So the MTBE on is kept low and the reverse reaction hardly occurs. Because of the distillation, almost all isobutylene can be converted into MTBE despite the unfavourable equilibrium, provided the catalyst bed is high enough. So by applying the principle of multifunctionality an equilibrium reaction can be forced to near completion in one pass.

Cumene has also been made by catalytic distillation, see Shoemaker and Jones (1987). Many aspects of the relevant vapour-liquid equilibria, the reaction and its kinetics have to be favourable in order that catalytic distillation can be applied successfully. Due to the presence of the vapour phase, the concentrations of some of the reactants in the liquid phase will be lower, thus reducing the reaction rate. For all but expensive catalysts and equipment, this reduction is more than off-set by the deletion of the separate separation step. Some more niches will be found for catalytic distillation, however, many preconditions have to be fulfilled, see also Mayer (1980):

- Temperature ranges for the distillation and the reaction must coincide.

- The reaction has to be fast enough so that the total residence time in the packed catalyst bed is sufficient.

- The reaction can not be so fast that the reaction occurs only in a small part of the bed, otherwise the reaction heat liberated only enhances the distillation locally.

- One of the desired products has to be either the highest or lowest boiling compound in the system, in order that by its separation, the equilibrium shifts in the desired direction, and of course, the reaction must be exothermic. An endothermic reaction would be quenched.

This process has also been recommended for the production of propylene glycol, see Kinoshita (1983) and for the separation of $\mathbf{m}$ - and p-xylene, see Saito (1971).

\section{Membrane reactors}

Membranes were originally developed for filtration. Dense non-porous membranes for hyperfiltration, also called reversed osmosis, and porous membranes used for ultrafiltration, with pore diameters of 3-200 nm, and for microfiltration, with pores of 0.2-10 microns. Polymeric membranes were almost exclusively limited to biotechnology, see e.g. Belfort (1989). We will restrict ourselves to inorganic membranes.

A distinction can also be made between dense and porous inorganic membranes. Foils of palladium or its alloys are permeable only to hydrogen and silver on dense zirconia only to oxygen. The selectivity of these dense membranes is, of course, very high, but permeation rates are very low. Costs limit the general application of these membranes.

Commercially available porous membranes are mostly based on alumina or zirconia. The membrane is at least 5 micron, but more usually 10-20 microns thick and placed on some intermediate support layers of a few $\mathrm{mm}$ thickness. Diffusion through the pores is of the Knudsen type, hence separation factors are related to the permeabilities of the diffusing compounts and to the square root of the ratio of their molar masses. Sometimes for very narrow pores separation factors are much higher, in what usually is called capillary condensation.

In the case of reactions, use is made of the membrane properties in several ways: In the case of equilibrium reactions, by removal of one of the products, so shifting the equilibrium in the desired direction. Side reactions can be suppressed by removal of products subject to decomposition. In cases where we wish to dose reactants slowly, membranes can give more precise control of the reaction. We will give a few examples of applications of inorganic membrane reactors, which, although receiving very much attention, but have not yet passed beyond the research and development stage. For a recent survey e.g. see Hsieh (1991).

In 1966, the first patent was granted for the use of $\mathrm{Pd}$ as a dense membrane reactor, in which hydrocarbons were dehydrogenated and the reaction rate increased by removal of the hydrogen, since then a great number of applications has been explored. For a recent survey, see Shu et al. (1991). A small scale pharmaceutical application was the synthesis of vitamin $K$ in one step from a mixture of 
quinone and acetic anhydride in a Pd-Ni membrane reactor with a yield of $95 \%$. This should be compared to the classical multisteps synthesis with a yield of $80 \%$, see Gryaznov (1986). Itoh et al. (1988) dehydrogenated cyclohexane in a $\mathrm{Pd}$ membrane reactor at $200^{\circ} \mathrm{C}$. At that temperature the equilibrium conversion of cyclohexane would be $19 \%$, but by continuous removal of $\mathrm{H}_{2}$ conversions above $99 \%$ were reached. Many applications have been tried. Selectivity is very high, but success on a large scale is hindered by the low fluxes through the dense membranes.

Porous membranes exhibit low selectivities but have much larger permeabilities. A recent survey was given by Armor (1989). A distriction must made whether the membrane is only a part of the reactor e.g. surrounding a catalyst bed or that the reaction takes place in the membrane itself. In the latter case the membrane is in most cases impregnated with a catalyst. Three examples follow.

Wu and Liu (1992) studied the dehydrogenation of ethylbenzene to styrene over a bed of catalyst pellets, surrounded by a ceramic membrane. $\mathrm{H}_{2}$ diffused more rapidly through the membrane than the aromatics. Usually this reaction is a.o. accompanied by hydrocracking of ethylbenzene to toluene and methane. By applying the membrane separation this side reaction was strongly suppressed and the styrene yield increased due to reduced $\mathrm{H}_{2}$ concentration in the reactor. Fluxes were however low.

Champagnie et al (1990) used Pt impregnated alumina membranes to increase the conversion of ethane to ethylene in the temperature range of $450-600^{\circ} \mathrm{C}$. They achieved conversions six times higher than the equilibrium conversion due to the selective removal of hydrogen. In catalyst-impregnated membranes reaction times are very short, hence only reactions with fast kinetics can be executed. Furthermore a typical problem emerges, namely the balance between reaction rates and diffusional permeation rates. The permeation rate of $\mathrm{H}_{2}$ in the above example must be larger that the dehydrogenation rate of ethane, in order to reduce the local hydrogen concentrations and so force the equilibrium in the desired direction. At the same time the permeation rate of ethane must be lower than the reaction rate. Usually there is a relatively narrow window of operation where conditions are favourable. This dehydrogenation of ethane was taking place with high fluxes.

For control of a reaction a membrane can be used to keep reactants separated, e.g. for partial combustion reactions in a porous, ceramic membrane. Reactants are fed to different sides and diffuse from either side into the membrane. A catalytically active material has been deposited in the pores. There is no pressure difference over the membrane. For fast reaction rates the permeation rates are limiting resulting in a small reaction zone somewhere in the membrane. For instantaneous, irreversible reactions the zone reduces to a reaction plane. Product(s) diffuse out of the membrane to both sides. The location of the reaction zone inside the membrane is such that the molar fluxes of the reactants are stoichiometrically equal. After an increase in the concentration of a reactant on either side of the membrane, the reaction zone will shift in such a way that the stoichiometry is again met, see Fig. 4 . Sloot et al. (1990) proved this experimentally for the Claus reaction:

$$
2 \mathrm{H}_{2} \mathrm{~S}+\mathrm{SO}_{2} \rightarrow 3 / 8 \mathrm{~S}_{8}+2 \mathrm{H}_{2} \mathrm{O}
$$
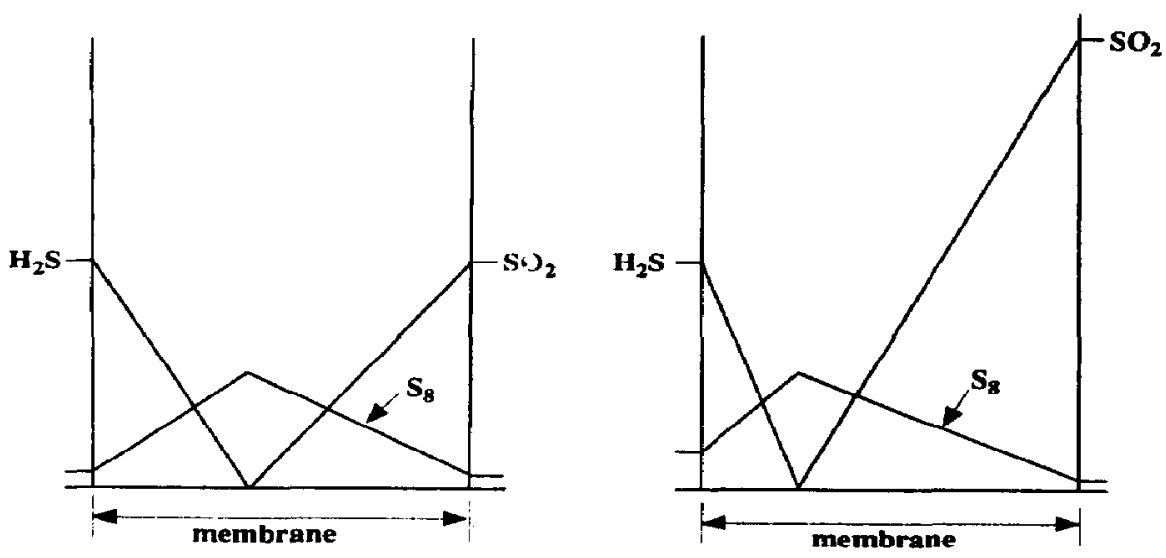

Fig. 4. Concentration profiles in a membrane for an instantaneous reaction. If a concentration at either side is changed, the reaction front moves inside the membrane 

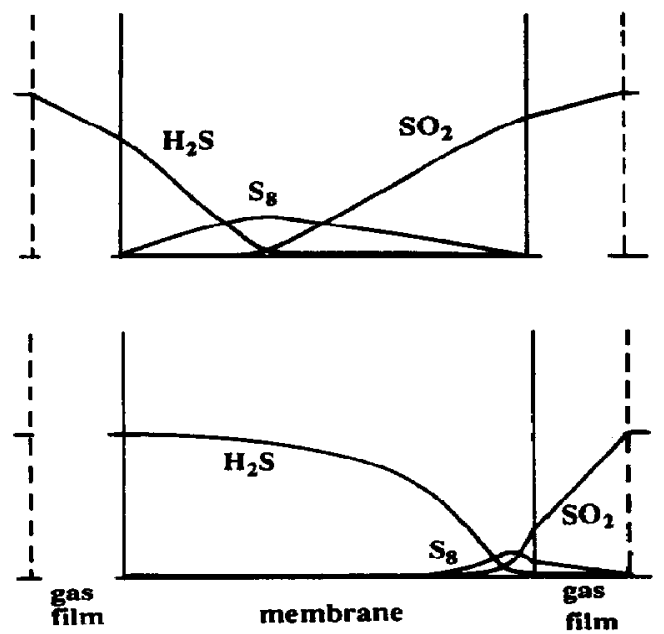

Fig. 5. Concentration profiles in a membrane for a fast reaction without (left) and with (right) a pressure difference over the membrane.

The drift of the sulphur to both sides of the membrane could be prevented by applying a pressure difference, so forcing the product in one direction, see Fig. 5. Fluxes were low.

Much work has also been done in the mathematical description of the behaviour of membrane reactors. Mohan and Govind $(1986,1988 \mathrm{a}, 1988 \mathrm{~b})$ published a set of papers in which, by simulation, they analyzed the influence of the important parameters, including: membrane characteristics and dimensions, reactor geometry and heat transfer rates, the pressures on both sides of the membrane, flow rates of the feed and the sweep gas, temperatures and concentrations in the feed, kinetics, equilibria, and reaction heats. Also permeability variations, recycle streams, and direction of the flow (being co- or countercurrent) were taken into account.

Membrane reactors are still under development and many problems still have to be solved. These include: the geometry of the reactor, the sealing and packing of the individual units -e.g. in the case of tube shaped membrane in a large array and under greatly varying thermal stresses-, and the fouling of membranes, e.g. by coke, for which steam has to be added. From the reaction engineering point of view major obstacles to their commercial introduction are:

- the low permeation rates. In most experimental studies until now the fluxes are still 10-1000 times too low to give a reactor volume with an acceptable capacity.

- the need for a sweep gas to remove the component(s) at the permeate side in order to keep concentrations low and consequently concentration differences over the membrane high, involves an additional separation step.

- the lack of an efficient method to supply or withdraw heat to or from membrane reactors on a large scale.

- the difficulty of keeping pressure differences across a membrane to almost zero, if desired, in large scale industrial reactors.

Much work still has to be done to tackle all these problems and we still have a long way to go. Some niche applications for membrane reactors surely will be found, the example mentioned for the vitamine K synthesis gives confidence.

Gas-Solid-Solid Trickle Flow Reactor (GSSTFR) and Reactor Section with Interstage Product Removal (RSIPR)

Equilibrium processes like the syntheses of ammonia and methanol require a cumbersome system to separate the product from the unconverted reactants and to recirculate these reactants to the reactor. Much energy is wasted in these separations and recirculations. Moreover, the feed preparation for these plants is complicated because a very pure feed of a stoichiometric compositions is required. Any impurity or reactant in excess, would accumulate in the recycle loop and slow down reaction rates and lower conversions. To overcome these problems Westerterp and Kuczynski (1987a, 1987b) developed two processes to achieve complete conversion in one pass through the reactor. In the gas-solid-solid trickle flow reactor GSSTFR they trickled a solid adsorbent countercurrently to the methanol synthesis gas through a fixed catalyst bed. The adsorbent selectively removed the methanol as it was formed. 


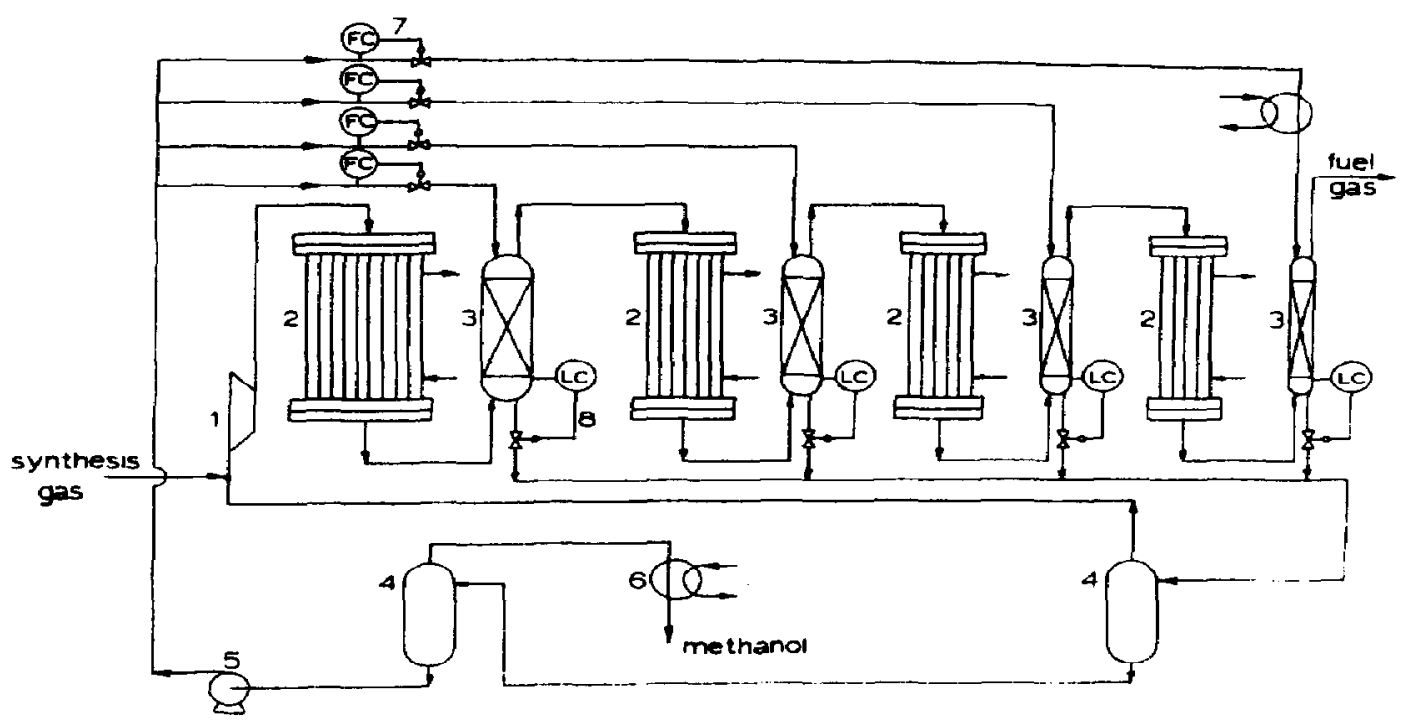

Fig. 6. The reactor section with interstage product removal for the methanol synthesis.

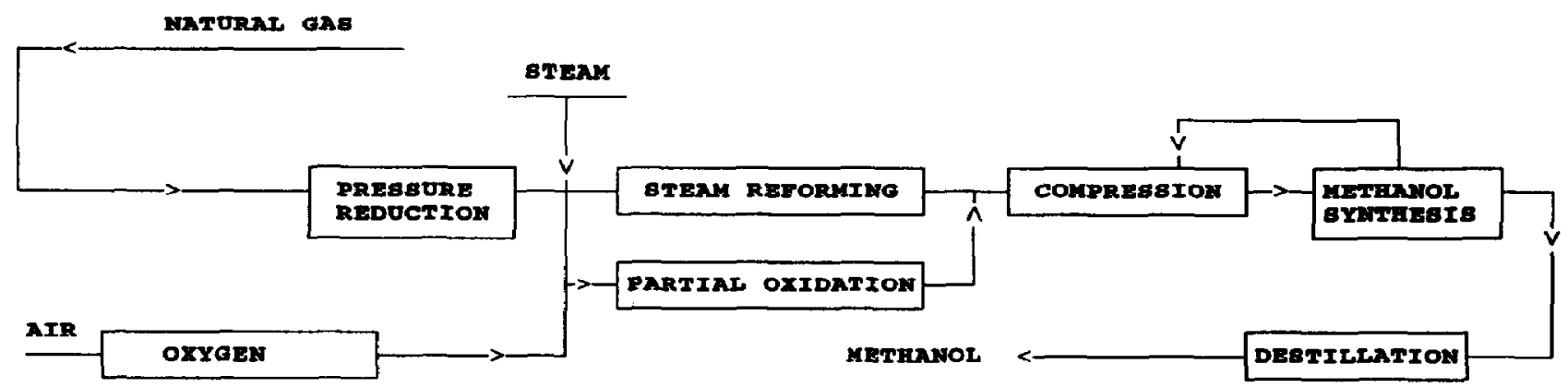

Fig. 7. The line-up of the units in a classical large scale methanol plant.

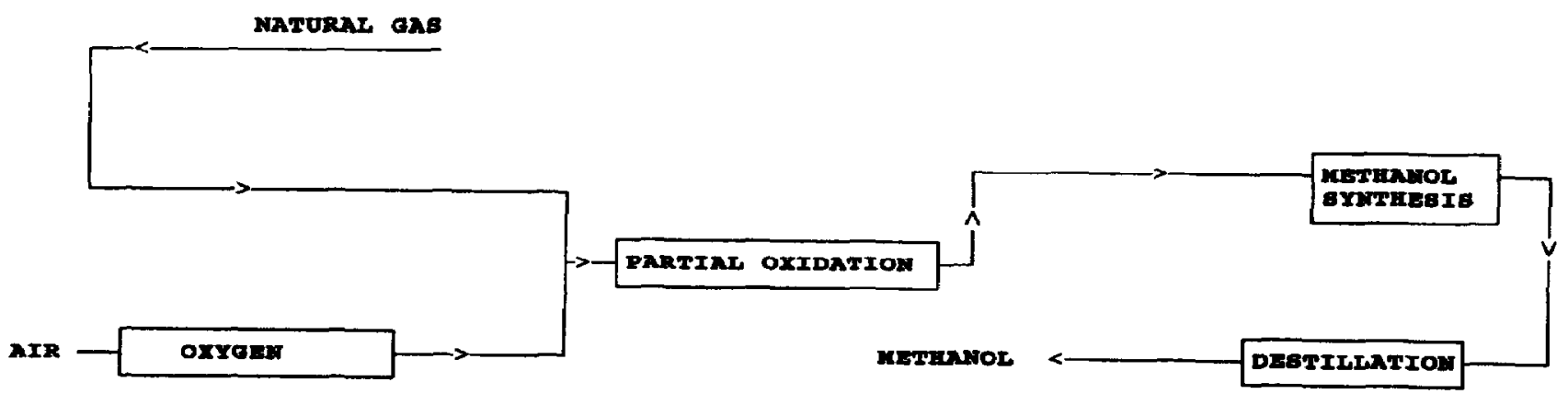

Fig. 8. The line-up of units in a methanol plant with a reactor section with interstage product removal. 
At high production rates they achieved $100 \%$ conversion and they ran a miniplant for 100 hours on a stoichiometric feed with the gas outlet closed and only a stream of solids, loaded with methanol leaving the reactor section.

To realise a process based on the GSSTFR on an industrial scale requires a cumbersome system for methanol desorption and solids recirculation. Therefore Westerterp et al. (1989) also developed a reactor section with interstage product removal RSIPR, based on absorption of methanol in a liquid solvent an the reaction temperature. Also this process was operated successfully on a miniplant scale. In four sets of reactors and absorbers $97-98 \%$ conversion can be achieved in a once-through operation without recycle, see Fig. 6. This RSIPR principle is based only in proven technology.

Although much energy is saved by applying the RSIPR process, its main strength lies in the fact it can also handle non-stoichiometric feeds. Any reactant in excess will pass through the reactor section as an inert material. An excess $\mathrm{CO}$ or $\mathrm{H}_{2}$ leaving the RSIPR can be used as fuel or for further processing in carbonylation, Fischer-Tropsh or hydroformylation processes and hydrogenations respectively. In Fig. 7 a block diagram shows the plants combined in a large methanol manufacturing unit. In the steam reforming unit, an excess of hydrogen, and in the partial oxidation unit, a feed stream deficient in hydrogen are produced and by careful balancing an exactly stoichiometric feed can be made.

Now in the case, where the stoichiometric requirement can be dropped, as in the RSIPR, we obtain the following simplifications, see Fig. 8. First no recycle is required anymore in the synthesis unit. Secondly the expensive steam reforming can be left out. As the partial oxidation is not an equilibrium process it also can be executed at higher pressures, say at synthesis pressure. Thirdly the expansion turbine and recompressor can be skipped and the natural gas, at well pressure, can be fed straight to the oxidation and synthesis unit. The excess $\mathrm{CO}$ can be used for other purposes, e.g. in a FischerTropsch unit. The oxidation unit delivers a stoichiometric ratio of $1.6-1.7$, for the methanol production we require 2.0 and for Fischer-Tropsch a ratio of 1.0 .

We observe that great savings can be obtained with the RSIPR principle applied to methanol synthesis, see also Westerterp (1992). The GSSTFR and RSIPR approaches can also be applied to consecutive reactions, by removing the desired product within the reactor.

\section{COMBINATION OF REACTIONS}

The combination of reactions in one single piece of equipment is also not new. In 1746 in England the so called lead chamber process was introduced to make sulphuric acid. In this process the oxidation of sulphur dioxide, according to $2 \mathrm{SO}_{2}+\mathrm{O}_{2}+2 \mathrm{H}_{2} \mathrm{O} \rightarrow 2 \mathrm{H}_{2} \mathrm{SO}_{4}$ was carried out simultaneously with the reduction: $2 \mathrm{NO}_{2} \rightarrow 2 \mathrm{NO}+\mathrm{O}_{2}$ and later in the process the $\mathrm{NO}$ was reoxidized to $\mathrm{NO}_{2}$ by air. We will present two examples of the application of multifuctionality to modern reactors.

Gryaznov et al. (1973) were the first to propose the coupling of two reactions in a membrane reactor, in which a hydrogenation and a dehydrogenation are carried out separated by the membrane. The exothermic reaction enthalpy of the hydrogenation reaction is used in the endothermic dehydrogenation. In a simulation study Itoh and Govind (1989) combined the dehydrogenation of butene- 1 with the (more obvious) combustion of hydrogen, which has permeated through a dense palladium membrane. In tubes which were coated with a palladium membrane dehydrogenation catalyst pellets were placed, around the tubes air was supplied to burn the hydrogen. They demonstrated that such a reactor can be operated autothermally and that reasonable conversion rates can be obtained. Maybe the combination of endoand exothermic reactions can solve the problems of heat supply or withdrawal in membrane reactors? At least, in reactors with dense membranes, it must also be possible to balance locally the heat absorption and production.

Recently Blanks et al. (1990) discussed the use of the adiabatic bed reactor with periodic flow reversal for a combination of an endo- and an exothermic reaction. Methane combustion with air $\mathrm{CH}_{4}+\mathbf{0 . 5}$ $\mathrm{O}_{2}+1.88 \mathrm{~N}_{2} \rightarrow 0.75 \mathrm{CH}_{4}+0.25 \mathrm{CO}_{2}+0.5 \mathrm{H}_{2} \mathrm{O}+1.88 \mathrm{~N}_{2}$ was combined with the endothermic steam and $\mathrm{CO}_{2}$ reforming process, $0.75 \mathrm{CH}_{4}+0.25 \mathrm{CO}_{2}+0.5 \mathrm{H}_{2} \mathrm{O} \rightarrow \mathrm{CO}+2 \mathrm{H}_{2}$, yielding a synthesis gas claimed to be suitable for Fischer-Tropsch syntheses. Of course, the water gas shift reaction $\mathrm{CO}+\mathrm{H}_{2} \mathrm{O} \rightarrow \mathrm{CO}_{2}+\mathrm{H}_{2}$ also occurred. They operated a miniplant and a pilot plant with a cylindrical reactor of $4 \mathrm{~m}$ length and diameter of $0.57 \mathrm{~m}$. For a catalyst, they employed a nickel reforming catalyst. Zones of inert material at inlet and outlet were used for the heating and cooling. Natural gas conversions from 85-97\%, and CO yields of 75-95\%, were obtained. Reactor capacities 
were around $2.5 \mathrm{kmol} / \mathrm{m}^{3} \mathrm{hr}$ and the process could be run autothermally, without external energy supply. Again a very promising result employing the principle of multifunctionality.

\section{CONCLUSIONS}

Few large scale applications of multifunctional reactors could be reported in the outline above. This should not discourage the reaction engineer nor make him pessimistic. Rather it should stimulate him to increase his endeavours to make them a success. The reactor with periodic flow reversal and the catalytic distillation reactor, have already found large scale applications. Dense membrane reactors may be the next. The application of the reactor section with interstage product removal to the methanol synthesis has shown us what great impact the application of the multifunctionality principle can have. Other multifunctional reactors are still rather far from large scale introduction.

We should realise that the more exotic our reactors become, the more delicate and difficult their construction and operation. This implies that an operator will not change to more complicated machinery for just minor savings in investments. Large advantages in operating costs must be envisaged. This will limit the rapid introduction of multifunctional reactors, despite their scientific and technological challenges. Last but not least we acknowledge the appeal of working on new, alternative and surprising concepts, yet want to stress that for many years much more of our effort in science must still be directed to a better and more profound understanding, and further development, of existing methods and technologies.

\section{REFERENCES}

Agar, D.W. and Ruppel, W., 1988, Extended reactor concept for dynamic DeNO design, Chem. Eng. Sci. 43, 2073-2078.

Agar, D.W. and Ruppel, W., 1988, Multifunktionale Reaktoren für die heterogene Katalyse, ChemieIng.-Tech. 60, 731-741.

Armor, J.N., 1989, Catalysis with permselective inorganic membranes, App. Catal. 49, 1-25

Belfort, G., 1989, Membranes and bioreactors: a technical challenge in biotechnology, Biotechnol. Bioeng. 33, 1047-1066.

Blanks, R.F., Wittrig, T.S. and Peterson, D.A., 1990, Bidirectional adiabatic synthesis gas generator, Chem. Eng. Sci. 45, 2407-2413.

Boreskov, G.K., Matros, Yu.Sh. and Kiselev, O.V., 1979, Catalytic processes carried out under nonstationary conditions, Kinet. Katal. 20, 773-780.

Champagnie, A.M., Tstotsis, T.T., Minet, R.G. and Webster, I.A., 1990, A high temperature catalytic membrane reactor for ethane dehydrogenation, Chem. Eng. Sci. 45, 2423-2429.

Coca, J. and Langer, S.H., 1983, Doing chemistry in the gas chromatograph, ChemTech. 13, 682-689.

Eigenberger, G. and Nieken, U., 1988, Catalytic combustion with periodic flow reversal, Chem. Eng. Sci. 42, 2109-2115.

Eigenberger, G. and Nieken, U., 1988, Abluftoxidation in Monolith-Katalysatoren mit periodischem Wechsel der Strömungsrichtung, Chem.-Ing.-Techn. 60, 1070-1071.

Eigenberger G. and Nieken, U., 1991, Katalytische Abluftreinigung: Verfahrenstechnische Aufgaben und neue Lösungen, Chem.-Ing.-Techn. 63, 781-791.

Fisch, B., Carr, R.W. and Aris, R., 1986, The continuous countercurrent moving bed chromatographic reactor, Chem. Eng. Sci. 41, 661-668.

Geelen, H. and Wijffels, J.B., 1965, The use of a distillation column as a chemical reactor, Proc. third Chem. React. Eng. Symposium, Pergamon, Oxford, 125-134.

Gelder, K.B. van, Damhof, J.K., Kroijenga, P.J. and Westerterp, K.R., 1990, Three-phase packed bed reactor with an evaporating solvent - I. Experimental: the hydrogenation of 2,4,6-trinitrotoluene in methanol, Chem. Eng. Sci. 45, 3159-3170.

Gelder, K.B. van, Borman, P.C., Weenink, R.J. and Westerterp, K.R., 1990, Three-phase packed bed reactor with an evaporating solvent - II. Modelling of the reactor, Chem. Eng. Sci. 45, 3171-3192.

Gryaznov, V.M., 1986, Hydrogen permeable palladium membrane catalysts. An aid to the efficient production of ultra pure chemicals and pharmaceuticals, Plat. Met Rev.30, 68-72. 
Gryaznov, V.M., Smirnov, V.S. and Slinko, G., 1973, Heterogeneous catalysis with reagent transfer through the selectively permeable catalyst, in Catalysis, J.W. Hightower ed., American Elsevier, New York.

Hsieh, H.P., 1991, Inorganic membrane reactors, Catal. Rev.-Sci. Eng. 33 (1 \& 2), 1-70.

Itoh, N. and Govind, R., 1989, Combined oxidation and dehydrogenation in a palladium membrane reactor, IEC Res. 28, 1554-1557.

Itoh, N., Shindo, Y., Haraya, K. and Hakuta, T., 1988, A membrane using microporous glass for shifting equilibrium of cyclohexane dehydrogenation, J. Chem. Eng. Japan 21, 399-404.

Kuczynski, M., Oyevaar, M.H., Pieters, R.T. and Westerterp, K.R., 1987, Methanol synthesis in a countercurrent gas-solid-solid trickle flow reactor. An experimental study, Chem. Eng. Sci. 42, 1887-1898.

Matros, Yu.Sh., 1985, Unsteady state processes in catalytic reactors, Elsevier, Amsterdam.

Matros, Yu.Sh., 1989, Catalytic processes under unsteady-state conditions, Elsevier, Amsterdam.

Mohan, K. and Govind, R., 1986, Analysis of a cocurrent membrane reactor, AIChE J. 32, 20832086.

Mohan, K. and Govind, R., 1988a, Analysis of equilibrium shift in isothermal reactors with a permselective wall, AIChE J. 34, 1493-1503.

Mohan, K. and Govind, R., 1988b, Effect of temperature on equilibrium shift reactors with a permselective wall, IEC Res. 27, 2064-2070.

Ray, A., Tonkevich, A.L., Aris, R. and Carr, R.W., 1990, The simulated countercurrent moving bed chromatographic reactor, Chem. Eng. Sci. 45, 2431-2437.

Saatdjian, E. and Large, J.F., 1985, Heat transfer simulation in a raining packed bed exchanger, Chem. Eng. Sci. 40, 693-697.

Schoenemann, K. and Hofmann, H., 1957, Die konsequente Anwendung der chemischen Reaktionstechnik, Chem.-Ing.-Techn. 29, 665-671.

Shoemaker, J.D. and Jones, E.M., 1987, Cumene by catalytic distillation, Hydroc. Process., June, 57-58.

Shu, J., Grandjean, B.P.A., van Neste, A. and Kaliaguine, S., 1991, Catalytic palladium-based membrane reactors: a review, Can. J. Chem. Engng. 69, 1036-1059.

Sloot, H.J., Versteeg, G.F. and van Swaaij, W.P.M., 1990, A non-permselective membrane reactor for chemical processes normally requiring strict stoichiometric feed rates of reactants, Chem. Eng. Sci. 45, 2415-2421.

Smith, L.A. and Huddleston, M.N., 1982, New MTBE design now commercial, Hydroc. Process., March, 121-123.

Westerterp, K.R. and Kuczynski, M., 1987, A model for a countercurrent gas-solid-solid trickle flow reactor for equilibrium reactions. The methanol synthesis, Chem. Eng. Sci. 42, 1871-1885.

Westerterp, K.R., Kuczynski, M. and Kamphuis, C.H.M., 1989, Synthesis of methanol in a reactor system with interstage product removal, IEC Research, 28, 763-771.

Westerterp, K.R., van Gelder, K.B., Janssen, H.J. and Oyevaar, M.H., 1988, Development of catalytic hydrogenation reactors for the fine chemicals industry, Chem. Eng. Sci. 43, 2229-2236. 